\title{
An Objective Way to Evaluate Continuity of Care in Residency
}

\author{
Brendan Kushneriuk MD \& Jason Hosain MD CCFP*
}

\begin{abstract}
Objective: To develop a tool to assess Canadian family medicine residents in regards to continuity of care (COC).

Design: Analysis of the first 100 patient visits of family medicine residents during their four-month block time in the second year of residency. Data was collected between the years of 2009 and 2012 and used to construct standardized curves for COC.

Setting: West Winds Primary Health Centre in Saskatoon, Saskatchewan.

Participants: 36 second-year family medicine residents training at West Winds Primary Health Centre in Saskatoon, Saskatchewan.

Main outcome measures: The number of unique patients that a family medicine second-year resident encounters within the first 100 patient visits of family medicine block time.

Results: Family medicine residents demonstrate individual variation in the number of unique patients they encounter within one hundred patient visits.

Conclusion: It is possible to develop a tool that can assess second-year family medicine residents in their ability to practise COC. This tool can be used to identify residents in difficulty, such that appropriate interventions can be made early on in their family medicine block time. Further research, involving residents from across Canada, is needed before this tool can be employed in a widespread manner.
\end{abstract}

Keywords: continuity of patient care, family practice, medical education, physician-patient relations, internship and residency, primary health care

Family medicine residents are subjected to a wide range of evaluations during their residency program. The College of Family Physicians of Canada (CFPC) has emphasized the Triple C. Competency-based Curriculum (Triple C), consisting of three main points: 1) comprehensiveness, 2) focus on continuity of education and patient care and 3) centered in family medicine (Kerr et al., 2011).

Continuity was described as 'continuity of care' (COC) by Hennen in 1975 and consists of four domains: chronological, geographical, interdisciplinary, and 
interpersonal (Delva, Kerr, \& Schultz, 2011). Since its initial introduction, the definition of $\mathrm{COC}$ has been drastically altered in response to changes within medical practice, since many family physicians have focused their practice on specific areas of care such as sports medicine, maternity, or hospital care (Delva et al., 2011; Bailey, 2007). Bell and Szafran (1995) described COC as "the provision of comprehensive, biopsychosocial medical care to a patient over time, during which a mutual attitudinal contract for care develops between the physician and the patient" ( $p$. 1881).

Although Triple C has strongly emphasized COC, it has been difficult to find ways to formally evaluate residents in COC, especially early on in their training (Bell \& Szafran, 1995). It is critical that there be "use of metrics in residency programs to measure and evaluate how effectively residents provide COC to their patients" (Kerr et al., 2011, p.1355). In order to properly educate residents, it would be ideal to have a tool that can objectively identify residents who are struggling in the area of $\mathrm{COC}$ early in their training. This tool would allow for early intervention, such that a successful outcome is more likely. We intend to create such a tool by developing standard curves that show differences among residents in regard to how many patients return to their care.

Consider the following two scenarios that illustrate the extremes of the $\mathrm{COC}$ spectrum. The first example is an emergency room physician in a large city. It can be expected that each patient this doctor sees is a new patient (Figure 1 ). This is an example of little or no COC. In contrast, a family physician with a full practice would see patients from the same group and would be expected to have the best possible COC (Figure2).

\section{Methods}

Ethics approval was obtained from the University of Saskatchewan's College of Medicine Research Ethics Committee (Med-REC) prior to commencement of this study. The Med Access electronic medical record was used to collect data from 36 second-year family medicine residents who trained at West Winds Primary Health Centre in Saskatoon, Saskatchewan between the years of 2009 to 2012. These residents were all graduates of a Canadian medical school. Residents had their first 100 patient visits during their second-year family medicine block time analyzed for the number of unique versus returning patients. This point in the residents' training was selected because it is the first block of continuous, uninterrupted time in the clinic when residents have no external impediment to continuity.

A unique patient is defined as a patient who the specific resident has not previously encountered.
Standardized curves were constructed and percentiles were calculated. The curves were constructed so that users of the tool could set a point where intervention is necessary. For example, a residency training coordinator could use the $25^{\text {th }}$ percentile line as a cut-off tool.

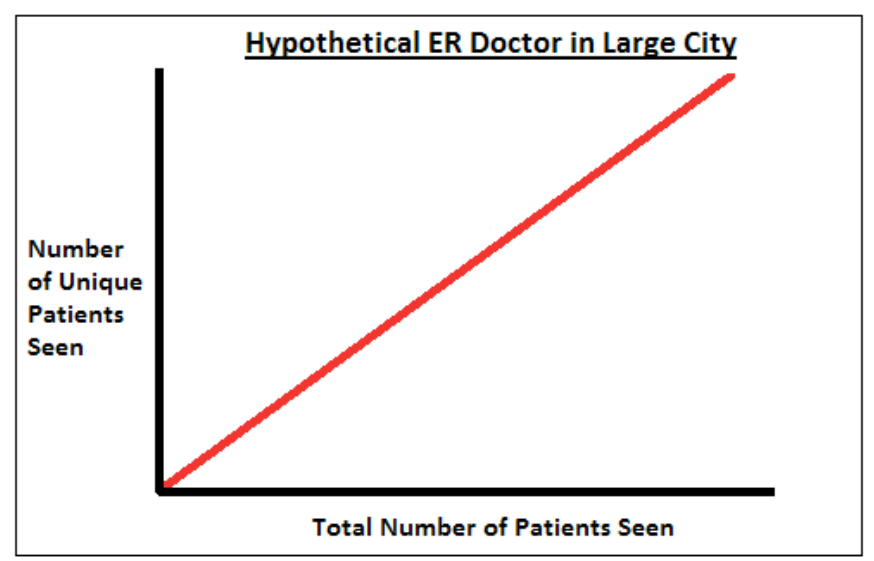

Figure 1: An example of the least COC expected in an emergency department in a large city. In this case, the slope is one because each patient coming through the door is expected to be a unique patient.

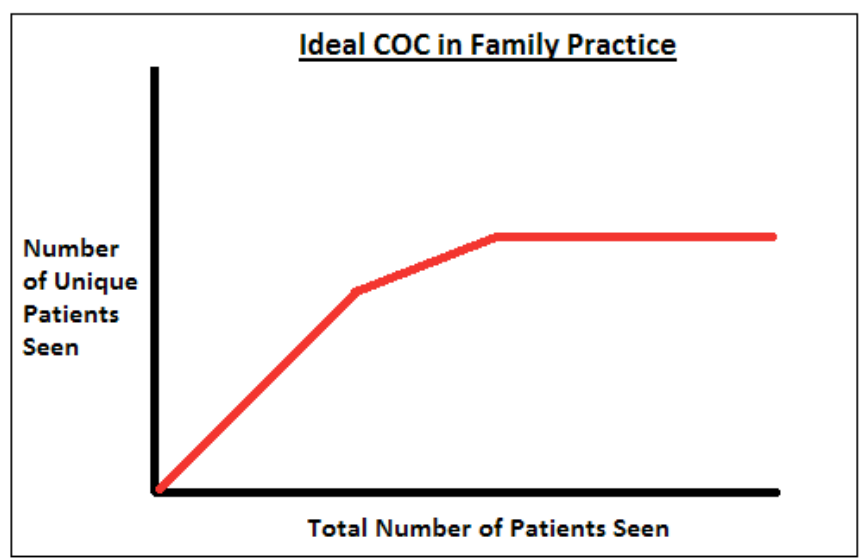

Figure 2: An example of good COC, seen in the office of a family physician that has closed his/her practice to new patients. Early on, the curve is steep because new patients are taken into the practice. However, when the practice closes, all patients have seen the physician before and the slope approaches zero.

\section{Results}

There was a sizeable difference in the number of unique patients seen by the 36 different residents. The mean number of unique patients seen was 86 and the median number was 87 . The range of unique patients seen was from 71 to 95 . The resident with 71 unique patients had the most $\mathrm{COC}$ and, in contrast, the resident with 95 unique visits had 
the least $\mathrm{COC}$.

Figure 3 shows percentiles obtained through analysis of the first 100 patient visits of the 36 family medicine residents. It is clear from Figure 3 that the percentile curves diverge by 100 patient visits. Following 100 patient visits, the $10^{\text {th }}$ percentile has seen 91 unique patients, while the $90^{\text {th }}$ percentile has seen 77 . This can be interpreted as indicating that the $10^{\text {th }}$ percentile has only examined 9 returning patients, while the $90^{\text {th }}$ percentile has examined 23 returning patients.
Figure 3 makes clear an apparent divergence of the standard curves after 100 patients, meaning there are measurable differences between individual family medicine residents in regards to $\mathrm{COC}$, and these differences can be identified early on in block time. Certain residents have fewer unique patients and more return visits, which correlates to Bell's definition of COC in terms of providing "biopsychosocial medical care to a patient over time" (Bell \& Szafran, 1995, p. 1880). Most residents are able to attain 100 patients visits within the first four weeks of family medicine block time, which is sixteen weeks in duration, so this is still

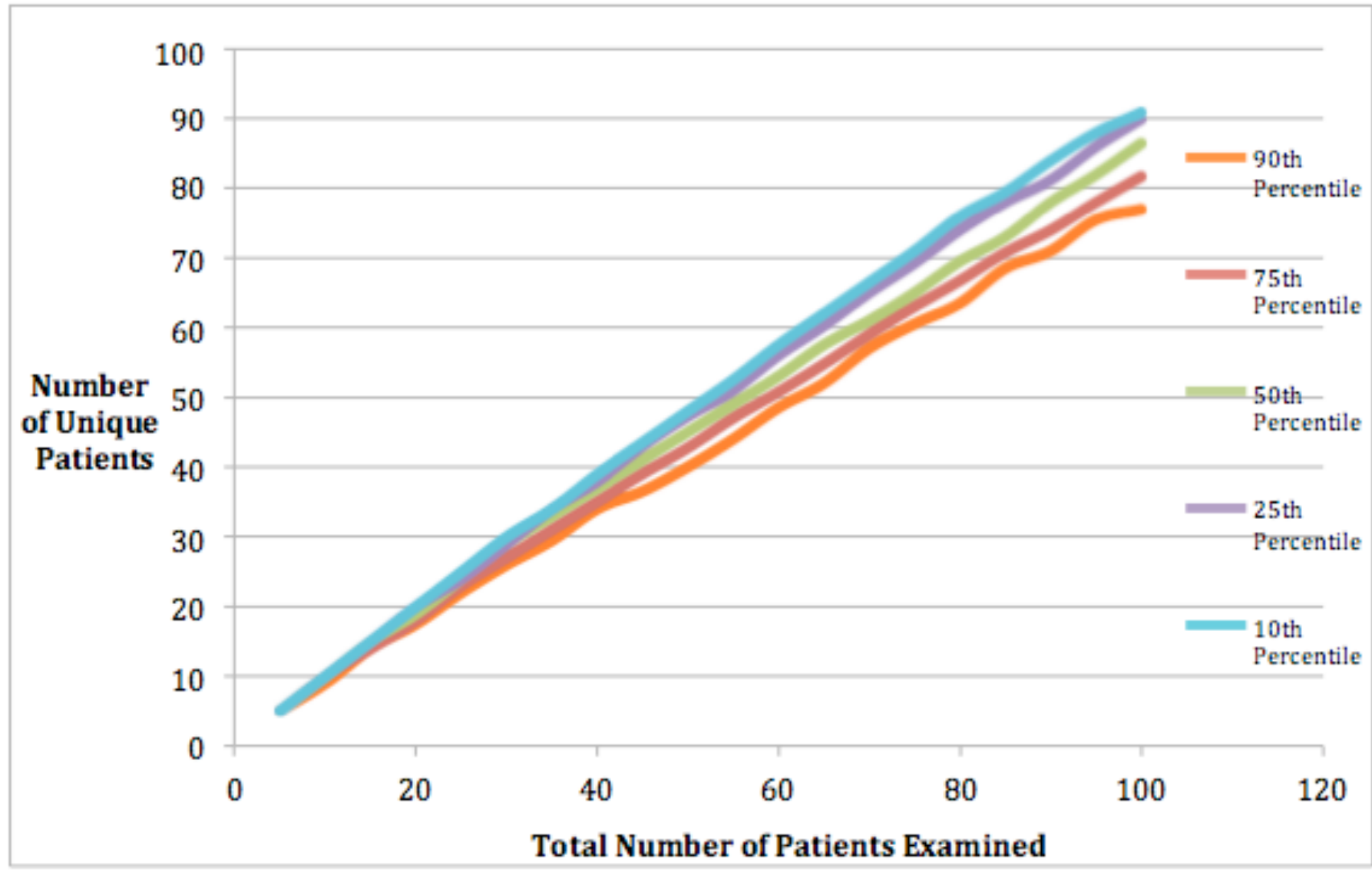

Figure 3: Number of unique patient visits in relation to total patient visits, labeled as percentiles.

\section{Discussion}

We used the first 100 patient encounters for this tool, rather than a time period, as different residents often see vastly different numbers of patients during their block time. Since our data set only considered the first 100 patient encounters, we cannot say with certainty that the curves will continue to diverge during block time, and it is possible that some of the residents who are identified by the tool could eventually establish good COC. However, as this is a screening tool and not a punitive tool, we feel that its high degree of sensitivity is reasonable. early in a resident's training. So that proper intervention can be made, ensuring a successful outcome, it is crucial this point be early in a residency. The purpose of devising this tool is not to penalize residents, but rather to help them succeed. It would be up to individual family medicine residency training programs to determine what percentile of COC constitutes adequate performance, and what intervention would be required, should a resident fall below that benchmark.

It is vital that objectivity be introduced into the process of evaluating family medicine residents in COC. This study has attempted to provide such an objective measure, 
but it is imperative to remember that this is only a tool. It does not replace the experience and judgment of residency training coordinators. This research represents a pilot project. Additional work must be done before this tool can be widely implemented. The sample size was not sufficiently large to make the percentile lines clearly divergent, so further work involving a larger data set will be necessary. The tool could also be made more robust by inclusion of residents from training sites across Canada. It is also important to note that the data used in this study came only from residents who did their undergraduate training in Canada and is not necessarily applicable to international medical graduates. Additional work could also be done to refine the tool, such that only chronic disease visits are considered. Finally, this work was done at a specific point in residency (i.e. second-year family medicine block time), so it is not clear whether this tool can be applied to other situations.

\section{Acknowledgements}

The authors would like to thank West Winds Primary Health Centre, for providing us with the data to analyze, as well as the University of Saskatchewan's College of Medicine Dean's Summer Student Research Projects, for funding the project. 


\section{Bibliography}

Bailey, T. (2007). The evolution of family medicine. Canadian Family Physician, 53(6), 1113 .

Bell, N. R., \& Szafran, O. (1995). Continuity of care. Opportunity for residents to see repeat patients. Canadian Family Physician, 41, 1880-1886.

Bice, T. W., \& Boxerman, S. B. (1977). A quantitative measure of continuity of care. Medical Care, 15(4), 347-349.

Campbell, M. K., Silver, R. W., Hoch, J. S., Østbye, T., Stewart, M., Barnsley, J. ... \& Tyrrell, C. (2005). Re-utilization outcomes and costs of minor acute illness treated at family physician offices, walk-in clinics, and emergency departments. Canadian Family Physician, 51, 82-83.

Delva, D., Kerr, J., \& Schultz, K. (2011). Continuity of care: Differing conceptions and values. Canadian Family Physician, 57(8), 915-921.

Fan, V. S., Burman, M., McDonell, M., \& Fihn, S. D. (2005). Continuity of care and other determinants of patient satisfaction with primary care. Journal of General Internal Medicine, 20(3), 226-233. doi: 10.1111/j.15251497.2005.40135.X

Hennen, B. K. (1975). Continuity of care in family practice. Part 1: dimensions of continuity. Journal of Family Practice, 2(5), 371-372.

Kerr, J., Walsh, A. E., Konkin, J., Tannenbaum, D., Organek, A. J., Parsons, E., ... \& Oandasan, I. (2011). Continuity: Middle (-a very good place to start. Canadian Family Physician, 57(11), 1355-1356

Merenstein, D., D'Amico, F., Devine, B., Mahaniah, K. J., Solomon, M., Reust, C. E., \& Rosenbaum, D. J. (2001). Longitudinal versus traditional residencies: A study of continuity of care. Family Medicine Journal, 33 (10), 746-750.
Rogers, J., \& Curtis, P. (1980). The concept and measurement of continuity in primary care. American Journal of Public Health, 70(2), 122127.

Salisbury, C., Sampson, F., Ridd, M., \& Montgomery, A. A. (2009). How should continuity of care in primary health care be assessed? British Journal of General Practice, 59(561), 134-41.

Turner, D., Tarrant, C., Windridge, K., Bryan, S., Boulton, M., Freeman, G., \& Baker, R. (2007). Do patients value continuity of care in general practice? An investigation using stated preference discrete choice experiments. Journal of Health Services \& Research Policy, 12 (3), 132-137. 
